\title{
Domain representability of $C_{\mathrm{p}}(X)$
}

by

\author{
Harold Bennett (Lubbock, TX) and David Lutzer (Williamsburg, VA)
}

\begin{abstract}
Let $C_{\mathrm{p}}(X)$ be the space of continuous real-valued functions on $X$, with the topology of pointwise convergence. We consider the following three properties of a space $X$ : (a) $C_{\mathrm{p}}(X)$ is Scott-domain representable; (b) $C_{\mathrm{p}}(X)$ is domain representable; (c) $X$ is discrete. We show that those three properties are mutually equivalent in any normal $T_{1}$-space, and that properties (a) and (c) are equivalent in any completely regular pseudo-normal space. For normal spaces, this generalizes the recent result of Tkachuk that $C_{\mathrm{p}}(X)$ is subcompact if and only if $X$ is discrete.
\end{abstract}

1. Introduction. Experience has shown that domain representability of a topological space, an idea borrowed from theoretical computer science, is a very strong completeness property in the sense of the Baire category theorem. For example, de Groot's subcompactness property [4] implies domain representability [1], and domain representability implies strong Choquet completeness, i.e., the existence of a winning strategy for the nonempty player in the strong Choquet game [7], a property that is much stronger than the familiar Baire space property "the intersection of countably many dense open sets is dense". Scott-domain representability is an even stronger completeness property. (Definitions appear in Section 2.)

Experience has also shown that it is difficult for the space $C_{\mathrm{p}}(X)$, of continuous real-valued functions on $X$ with the topology of pointwise convergence, to have strong completeness properties. While $C_{\mathrm{p}}(X)$ can be a Baire space [5], it rarely has the stronger completeness properties mentioned above. For example, it was recently proved that for any completely regular $X, C_{\mathrm{p}}(X)$ is subcompact if and only if $X$ is discrete, thereby answering an old question from the folklore [6]. Knowing that every subcompact regular

2000 Mathematics Subject Classification: Primary 54C35; Secondary 54E52, 06B35, $05 \mathrm{~F} 30$.

Key words and phrases: domain, Scott domain, Scott topology, domain representable space, pointwise convergence topology, normal space, pseudo-normal space, subcompact space, Choquet complete. 
space is domain representable and inspired by Tkachuk's methods in [6], we began to ask whether $C_{\mathrm{p}}(X)$ could be domain representable, except in the trivial case where $X$ is discrete. In this paper, we settle that question for normal spaces and we generalize the main result of [6] for normal spaces, by proving:

Main TheOREM. The following properties of a normal space are equivalent:

(a) $C_{\mathrm{p}}(X)$ is Scott-domain representable;

(b) $C_{\mathrm{p}}(X)$ is domain representable;

(c) $X$ is discrete.

The theorem is proved in Section 3. Normality plays a key role in some of the lemmas used in the proof and we do not know whether our result holds without normality. However, as we prove in the paper's final section, parts (a) and (c) of our main theorem are equivalent in any pseudo-normal, completely regular space.

Throughout this paper, all spaces are at least Hausdorff. We reserve the symbol $\mathbb{R}$ for the usual space of real numbers. Cardinals are initial ordinals, and for any ordinal $\beta,|\beta|$ is the first cardinal that is less than or equal to $\beta$.

2. Definitions and preliminary results. A particularly good introduction to the use of domain theory is given by Martin, Mislove, and Reed in [8] and we generally follow their terminology and notation. The supremum of a subset $S$ of a partially ordered set $(P, \sqsubseteq)$ is an upper bound $u$ for $S$ in $P$ that has $u \sqsubseteq v$ for each upper bound $v$ for $S$. A nonempty subset $D \subseteq P$ is directed provided that for each pair $d_{1}, d_{2} \in D$, some $d_{3} \in D$ has $d_{1}, d_{2} \sqsubseteq d_{3}$. A partially ordered set is a directed complete partial order (dcpo) if each nonempty directed subset of $P$ has a supremum in $P$. In a dcpo, Zorn's lemma shows that for each $x \in P$, there is a maximal member of $p \in P$ with $x \sqsubseteq p$, and the set of all maximal members of $P$ is denoted by $\max (P)$.

Given two elements $p, q$ of a poset $(P, \sqsubseteq)$, we define $p \ll q$ to mean that for any directed set $D$ with $q \sqsubseteq \sup (D)$, some $d \in D$ has $p \sqsubseteq d$. For $p, q \in P$ we use the notations $\Downarrow(q)=\{x \in P: x \ll q\}$ and $\Uparrow(p)=\{x \in P: p \ll x\}$, and the notations $\uparrow(p)=\{x \in P: p \sqsubseteq x\}$ and $\downarrow(q)=\{x \in P: x \sqsubseteq q\}$.

To say that a poset $P$ is continuous means that for each $p \in P$, the set $\Downarrow(p)$ is directed and has $p=\sup (\Downarrow(p))$. A domain is a continuous dcpo, and a Scott domain is a domain with the additional property that if $p, q \in P$ and $\uparrow(p) \cap \uparrow(q) \neq \emptyset$, then $\sup (p, q) \in P$. The following Interpolation Lemma is a key tool in domain theory [8]. 
Lemma 2.1. Suppose $(P, \sqsubseteq)$ is a domain and $a, c \in P$ with $a \ll c$. Then there is some $b \in P$ with $a \ll b \ll c$.

Lemma 2.2. Suppose $(P, \sqsubseteq)$ is a domain and $p, q, r \in P$ with $p \in \Uparrow(q) \cap$ $\Uparrow(r)$. Then there is an $s \in P$ with $p \in \Uparrow(s) \subseteq \Uparrow(q) \cap \Uparrow(r)$ and $s \ll q$ and $s \ll r$.

Proof. We note that $q$ and $r$ belong to the directed set $\Downarrow(p)$ so there is some $s^{\prime} \in \Downarrow(p)$ with $q, r \sqsubseteq s^{\prime} \ll p$. Apply the Interpolation Lemma 2.1 to find some $s \in P$ with $s^{\prime} \ll s \ll p$.

Given a domain $P$, the collection $\{\Uparrow(p): p \in P\}$ is the basis for a topology on $P$ that is called the Scott topology. To say that a topological space $X$ is (Scott-) domain representable means that there is a (Scott) domain $(P, \sqsubseteq)$ with the property that $\max (P)$, topologized using the relative Scott topology of $P$, is homeomorphic to $X$.

Basic open neighborhoods of a function $f$ in the topology of $C_{\mathrm{p}}(X)$ have the form $O(f, S, \varepsilon)=\left\{g \in C_{\mathrm{p}}(X):|g(x)-f(x)|<\varepsilon\right.$ for all $\left.x \in S\right\}$, where $\varepsilon>0$ and $S$ is a finite subset of $X$. Basic open neighborhoods of $f \in \max (P)=C_{\mathrm{p}}(X)$ have the form $\Uparrow(p) \cap \max (P)$, where $p \in P$ and $p \ll f$. The interplay of these two kinds of sets will be crucial in the proof of our main theorem.

Suppose we have a completely regular space $X$ and a continuous $f$ : $X \rightarrow \mathbb{R}$. It is possible to find a continuous function that agrees with $f$ on $S$ and has pre-assigned values at finitely many other points. More precisely, given any closed set $S \subseteq X$, any finite subset $T=\left\{y_{1}, \ldots, y_{n}\right\} \subseteq X-S$, and any sequence $r_{1}, \ldots, r_{n} \in \mathbb{R}$, there is a continuous $g: X \rightarrow \mathbb{R}$ with $g(x)=f(x)$ for each $x \in S$ and $g\left(y_{k}\right)=r_{k}$. In some of the lemmas to be proved in Section 4 below, we need even more. We will have a continuous function $f: X \rightarrow \mathbb{R}$ and a closed set $S$, and we will want to modify $f$ on a countable, closed, discrete set of points $\left\{z_{n}: n<\omega\right\} \subseteq X-S$. As the Tikhonov plank and the space of Problem (5I) in [3] show, complete regularity of $X$ is not enough to allow us to do that. Therefore, our lemmas will deal with completely regular pseudo-normal spaces, i.e., spaces with the property that given two disjoint closed sets $C, D$, one of which is countable, there are disjoint open sets $U, V$ with $C \subseteq U$ and $D \subseteq V$. For such spaces we have:

Lemma 2.3. Suppose $X$ is completely regular and pseudo-normal. Suppose $S$ is a closed subset of $X$ and that $u \in C_{\mathrm{p}}(X)$. If $\left\{z_{n}: n<\omega\right\} \subseteq X-S$ is a closed, discrete subset of $X$ and if $\left\{r_{n}: n<\omega\right\}$ is any set of real numbers, then there is a function $u_{1} \in C_{\mathrm{p}}(X)$ with $u_{1}(x)=u(x)$ for each $x \in S$ and $u_{1}\left(z_{n}\right)=r_{n}$. 
Proof. Because $X$ is regular and pseudo-normal, there is a discrete collection $\left\{W_{n}: n<\omega\right\}$ of open subsets of $X$ with $z_{n} \in W_{n} \subseteq X-S$. Using complete regularity, for each $n$ find a function $h_{n} \in C_{\mathrm{p}}(X)$ with $h_{n}(x)=0$ for each $x \in X-W_{n}$ and $h_{n}\left(z_{n}\right)=r_{n}-u\left(z_{n}\right)$. Because the collection $\left\{W_{n}: n<\omega\right\}$ is discrete, the function $h=\sum\left\{h_{n}: n<\omega\right\}$ belongs to $C_{\mathrm{p}}(X)$. Hence so does the function $u_{1}=u+h$ and we see that $u_{1}$ has the required properties.

Our next result seems to be part of the folklore of domain representability. The heart of the lemma is in Section I-2 of [2].

Lemma 2.4. Suppose the space $X_{i}$ is representable as $\max \left(P_{i}\right)$ for some $(S \operatorname{cott})$ domain $\left(P_{i}, \sqsubseteq_{i}\right)$ for each $i \in I$. We may assume that $\min \left(X_{i}\right) \in P_{i}$ for each $i \in I$. Then the coordinatewise ordered product set $\prod\left\{P_{i}: i \in I\right\}$ is a (Scott) domain and represents the Tikhonov product space $\prod\left\{X_{i}: i \in I\right\}$. In particular, for any cardinal $\kappa$, the product space $\mathbb{R}^{\kappa}$ is Scott-domain representable.

Proof. Let $\ll_{i}$ denote the auxiliary ordering of the poset $\left(P_{i}, \sqsubseteq_{i}\right)$. If $\left(P_{i}, \sqsubseteq_{i}\right)$ has a minimum element, call it $\theta_{i}$, and if $P_{i}$ does not have a minimum element, add one called $\theta_{i}$ lying below every other element of $P_{i}$. Exercise I-2-18 of [2] shows that the set $P:=\prod\left\{P_{i}: i \in I\right\}$ with the coordinatewise partial order $\sqsubseteq$ is a domain, and it is easy to check that if each $P_{i}$ is a Scott domain, then so is $P$. A key feature of $P$ (proved in the previously cited exercise) is that if $\left\langle a_{i}: i \in I\right\rangle,\left\langle b_{i}: i \in I\right\rangle$ are points of $P$, then in the auxiliary order $\ll$ on $P$, we have

(*) $\left\langle a_{i}\right\rangle \ll\left\langle b_{i}\right\rangle$ if and only if $a_{i} \ll_{i} b_{i}$ for each $i \in I$ and $\left\{i \in I: a_{i} \neq \theta_{i}\right\}$ is finite.

Clearly, there is a natural mapping between $\max (P)$ and $\prod\left\{\max \left(P_{i}\right)\right.$ : $i \in I\}=\prod\left\{X_{i}: i \in I\right\}$, and assertion $(*)$ shows that under that natural mapping, if $\left\langle a_{i}\right\rangle \in P$, then $\Uparrow\left(\left\langle a_{i}\right\rangle\right) \cap \max (P)=\prod\left\{\Uparrow\left(a_{i}\right) \cap X_{i}: a_{i} \neq \theta_{i}\right\} \times$ $\prod\left\{X_{i}: a_{i}=\theta_{i}\right\}$. Therefore the Scott topology on $\max (P)$ coincides with the product topology on $\prod\left\{X_{i}: i \in I\right\}$.

Finally, recall that $\mathbb{R}$ is locally compact, so that $\mathbb{R}$ is Scott-domainrepresentable, and now apply the first part of this lemma to conclude that each product space $\mathbb{R}^{\kappa}$ is also Scott-domain representable, as claimed.

3. Proof of the main theorem. As announced in Section 1, the main theorem of our paper asserts that for any normal space, the following three properties are equivalent: (a) $C_{\mathrm{p}}(X)$ is Scott-domain representable; (b) $C_{\mathrm{p}}(X)$ is domain representable; (c) $X$ is discrete. Clearly, (a) implies (b). Also, (c) implies (a) because if $X$ is discrete, then $C_{\mathrm{p}}(X)$ is the full topological product space $\mathbb{R}^{X}$, which is known to be Scott-domain representable 
by Lemma 2.4. Therefore it suffices to prove that (b) implies (c). Our proof will quote a sequence of lemmas whose proofs appear in the next section of the paper.

For contradiction, suppose $X$ is not discrete and yet $C_{\mathrm{p}}(X)$ is domain representable using a domain $(P, \sqsubseteq)$. Because $X$ is not discrete, there are nonclosed subsets of $X$. Let $\kappa$ be the least cardinal of a nonclosed subset of $X$. Then any subset of $X$ with cardinality $<\kappa$ is closed and discrete, and Lemma 4.1 shows that $\kappa>\omega$. Fix a subset $Y \subseteq X$ that has cardinality $\kappa$ and is not closed in $X$. Fix $z \in \operatorname{cl}(Y)-Y$. Well order $Y$ as $Y=\left\{y_{\alpha}: \alpha<\kappa\right\}$.

Because $(P, \sqsubseteq)$ is a domain that represents $C_{\mathrm{p}}(X)$, we know that when $\max (P)$ is endowed with the relative Scott topology, $\max (P)$ is homeomorphic to $C_{\mathrm{p}}(X)$. We will abuse notation and write $\max (P)=C_{\mathrm{p}}(X)$.

We will say that a 4 -tuple $(\beta, D, E, S)$ is acceptable if

(A1) $\beta$ is an ordinal with $\beta<\kappa, D \cup E \subset P$, and $\{z\} \cup\left\{y_{\alpha}: \alpha<\beta\right\} \subseteq$ $S \subseteq X$

(A2) $|D \cup E \cup S| \leq \max (|\beta|, \omega)$;

(A3) $D$ and $E$ are both bounded subsets of $P$;

(A4) if $d \in D$ (respectively $e \in E$ ) then some $\widehat{d} \in D$ has $d \ll \widehat{d}$ (resp., some $\widehat{e} \in E$ has $e \ll \widehat{e})$;

(A5) if $u_{1}, u_{2} \in \max (P)=C_{\mathrm{p}}(X)$ with $D \subseteq \downarrow\left(u_{1}\right) \cap \downarrow\left(u_{2}\right)$ (respectively, with $\left.E \subseteq \downarrow\left(u_{1}\right) \cap \downarrow\left(u_{2}\right)\right)$, then $u_{1}(x)=u_{2}(x)$ for each $x \in S$;

(A6) if $u_{1}, u_{2} \in \max (P)$ with $D \subseteq \downarrow\left(u_{1}\right)$ (respectively, with $E \subseteq \downarrow\left(u_{1}\right)$ ) and if $u_{2}(x)=u_{1}(x)$ for each $x \in S$, then $D \subseteq \downarrow\left(u_{2}\right)$ (resp., $\left.E \subseteq \downarrow\left(u_{2}\right)\right)$;

(A7) if $u, v \in \max (P)$ have $D \subseteq \downarrow(u)$ and $E \subseteq \downarrow(v)$, then $u(z)+v(z)=1$ (where $z$ is the limit point of $Y$ chosen above), and if $x \in S-\{z\}$, then $u(x)+v(x)=0$.

We will say that an acceptable 4-tuple $(\beta, D, E, S)$ is fully acceptable provided the sets $D$ and $E$ are directed subsets of $P$. Recall that any directed subset of a domain $P$ is a bounded subset of $P$. Consequently, as the names suggest, any fully acceptable 4-tuple is also an acceptable 4-tuple. Because constructing directed sets, rather than just bounded sets, is a challenge, we will need both of these ideas in our paper.

Let $\Psi$ denote the collection of all fully acceptable 4 -tuples and partially order $\Psi$ by the rule that $\left(\beta_{1}, D_{1}, E_{1}, S_{1}\right) \preceq\left(\beta_{2}, D_{2}, E_{2}, S_{2}\right)$ if and only if either $\left(\beta_{1}, D_{1}, E_{1}, S_{1}\right)=\left(\beta_{2}, D_{2}, E_{2}, S_{2}\right)$ or else $\beta_{1}<\beta_{2}$ and $D_{1} \subseteq D_{2}$, $E_{1} \subseteq E_{2}$, and $S_{1} \subseteq S_{2}$. Corollary 4.6 shows that there is a fully acceptable 4-tuple $\left(1, D^{*}, E^{*}, S^{*}\right)$. Hence $\Psi \neq \emptyset$. The Hausdorff Maximal Principle then yields a maximal chain $\Phi \subseteq \Psi$.

Let $I=\pi_{1}[\Phi]$, and write $\Phi=\left\{\left(\beta, D_{\beta}, E_{\beta}, S_{\beta}\right): \beta \in I\right\}$. Then by Lemma 4.7 we know that $I$ is cofinal in $\kappa$. Let $\widehat{D}=\bigcup\left\{D_{\beta}: \beta \in I\right\}, \widehat{E}=\bigcup\left\{E_{\beta}\right.$ : 
$\beta \in I\}$ and $\widehat{S}=\bigcup\left\{S_{\beta}: \beta \in I\right\}$. Then $\widehat{D}$ and $\widehat{E}$ are directed subsets of $P$, because each is the union of a chain of directed subsets of $P$. Therefore $\sup (\widehat{D})$ and $\sup (\widehat{E})$ exist in $P$, so there are elements $u, v \in \max (P)=C_{\mathrm{p}}(X)$ with $\widehat{D} \subseteq \downarrow(u)$ and $\widehat{E} \subseteq \downarrow(v)$.

Consider any $y_{\alpha} \in Y$. Because $I$ is cofinal in $\kappa$ we may choose $\beta \in I$ with $\alpha<\beta$. Then $y_{\alpha} \in S_{\beta}$. Because $D_{\beta} \subseteq \widehat{D} \subseteq \downarrow(u)$ and $E_{\beta} \subset \widehat{E} \subseteq \downarrow(v)$, property (A7) of the fully acceptable 4 -tuple $\left(\beta, D_{\beta}, E_{\beta}, S_{\beta}\right)$ guarantees that $u\left(y_{\alpha}\right)+v\left(y_{\alpha}\right)=0$ because $\alpha<\kappa$. At the same time, we have $u(z)+v(z)=1$ and that is impossible because $u+v$ is continuous and $z$ is a limit point of the set $Y=\left\{y_{\alpha}: \alpha<\kappa\right\}$. Therefore, modulo proving the lemmas used above, our main theorem is established.

4. Lemmas for the main theorem. This section is devoted to a series of results that combine to give the lemmas used in the proof of our main theorem in the previous section. Some of the lemmas are proved under the hypothesis that $X$ is completely regular and pseudo-normal (see Section 2) and the lemmas proved under this weaker hypothesis will be used in our paper's final section. Several of our lemmas, however, seem to require the full power of normality, which accounts for the hypothesis of normality in the main theorem stated in Section 1.

All lemmas in this section use the notation developed in Sections 2 and 3. Our first lemma is a baby version of the arguments used later in this section, and it might be helpful for the reader to see what happens without all of the general machinery to be developed later.

Lemma 4.1. If $X$ is completely regular and $C_{\mathrm{p}}(X)$ is domain representable, then every countable subset of $X$ is closed. Hence $\kappa>\omega$.

Proof. Let $(P, \sqsubseteq)$ be a domain that represents $C_{\mathrm{p}}(X)$. Suppose there is a countable subset $Y=\left\{y_{n}: n<\omega\right\}$ of $X$ that is not closed. Let $z \in \operatorname{cl}(Y)-Y$. Because $X$ is completely regular, there is a function $u_{0} \in$ $C_{\mathrm{p}}(X)$ with $u_{0}\left(y_{0}\right)=0$ and $u_{0}(z)=1$. The set $O\left(u_{0},\left\{y_{0}, z\right\}, 1\right)$ is a neighborhood of $u_{0}$ in $C_{\mathrm{p}}(X)=\max (P)$. Then there is a point $p_{0} \in P$ with $u_{0} \in \Uparrow\left(p_{0}\right) \cap \max (P) \subseteq O\left(u_{0},\left\{y_{0}, z\right\}, 1\right)$. There is a finite set $S_{0} \subseteq X$ and a number $\varepsilon_{0} \in(0,1)$ with $u_{0} \in O\left(u_{0}, S_{0}, \varepsilon_{0}\right) \subseteq \Uparrow\left(p_{0}\right) \subseteq O\left(u_{0},\left\{y_{0}, z\right\}, 1\right)$. Then $\left\{y_{0}, z\right\} \subseteq S_{0}$. Because $X$ is completely regular, there is a function $v_{0} \in C_{\mathrm{p}}(X)$ with $v_{0}(z)=0$ and $v_{0}(x)=-u_{0}(x)$ for all $x \in S_{0}-\{z\}$. Then $O\left(v_{0}, S_{0}, \varepsilon_{0}\right)$ is a neighborhood of $v_{0}$ in $C_{\mathrm{p}}(X)=\max (P)$ so there is a $q_{0} \in P$ with $v_{0} \in \Uparrow\left(q_{0}\right) \cap \max (P) \subseteq O\left(v_{0}, S_{0}, \varepsilon_{0}\right)$. Find a finite set $T_{0}$ and a positive $\eta_{0}$ with $O\left(v_{0}, T_{0}, \eta_{0}\right) \subseteq \Uparrow\left(q_{0}\right) \cap \max (P) \subseteq O\left(v_{0}, S_{0}, \varepsilon_{0}\right)$. Then $S_{0} \subseteq T_{0}$ and $\eta_{0} \leq \varepsilon_{0}$.

Because $X$ is completely regular and $T_{0}$ is finite, there is a function $u_{1} \in C_{\mathrm{p}}(X)$ with $u_{1}(x)=u_{0}(x)$ for each $x \in S_{0}$ and $u_{1}(x)=-v_{0}(x)$ for 
each $x \in T_{0}-S_{0}$. Because $S_{0} \subseteq T_{0}$ and $\eta_{0} \leq \varepsilon_{0}$ we have

$$
O\left(u_{1}, T_{0}, \eta_{0}\right) \subseteq O\left(u_{1}, S_{0}, \varepsilon_{0}\right)=O\left(u_{0}, S_{0}, \varepsilon_{0}\right) \subseteq \Uparrow\left(p_{0}\right)
$$

so that we can find some $p_{1} \in P$ with $u_{1} \in \Uparrow\left(p_{1}\right) \cap \max (P) \subseteq O\left(u_{1}, T_{0}, \eta_{0}\right) \subseteq$ $\Uparrow\left(p_{0}\right)$. Lemma 2.2 allows us to assume that $p_{0} \ll p_{1}$. Next we find a finite set $S_{1}$ and a number $\varepsilon_{1} \in\left(0,2^{-1}\right)$ with $O\left(u_{1}, S_{1}, \varepsilon_{1}\right) \subseteq \Uparrow\left(p_{1}\right) \cap \max (P) \subseteq$ $O\left(u_{1}, T_{0}, \eta_{0}\right)$. Then $T_{0} \subseteq S_{1}$ and $\varepsilon_{1} \leq \eta_{0}$. In addition, we may expand the set $S_{1}$ if necessary to ensure that $y_{1} \in S_{1}$.

Because $X$ is completely regular, there is a function $v_{2} \in C_{\mathrm{p}}(X)$ such that $v_{2}(x)=v_{1}(x)$ if $x \in T_{0}$ and with $v_{2}(x)=-u_{1}(x)$ for each $x \in S_{1}-T_{0}$. Then there is some $q_{1} \in P$ with $v_{1} \in \Uparrow\left(q_{1}\right) \cap \max (P) \subseteq O\left(v_{1}, S_{1}, \varepsilon_{1}\right) \subseteq$ $O\left(v_{0}, T_{0}, \eta_{0}\right) \subseteq \Uparrow\left(q_{0}\right)$. Lemma 2.2 allows us to assume that $q_{0} \ll q_{1}$. Then there is a finite set $T_{1}$ and a positive $\eta_{1}$ with $O\left(v_{1}, T_{1}, \eta_{1}\right) \subseteq \Uparrow\left(q_{1}\right) \cap \max (P) \subseteq$ $O\left(v_{1}, S_{1}, \varepsilon_{1}\right)$ so that $S_{1} \subseteq T_{1}$ and $\eta_{1} \leq \varepsilon_{1}$. Note that if $x \in S_{1}$ with $x \neq z$, then $u_{1}(x)+v_{1}(x)=0$, while $u_{1}(z)+v_{1}(z)=1$.

This recursion continues, producing a sequence of finite sets $\left\{y_{0}, z\right\} \subseteq$ $S_{0} \subseteq T_{0} \subseteq S_{1} \subseteq T_{1} \subseteq \cdots$ with $y_{k} \in S_{k}$, functions $u_{k}, v_{k} \in C_{\mathrm{p}}(X)$, elements $p_{0} \ll p_{1} \ll \cdots$ and $q_{0} \ll q_{1} \ll \cdots$ of the domain $P$, and positive numbers $\eta_{k} \leq \varepsilon_{k}<2^{-k}$ with $O\left(u_{k}, S_{k}, \varepsilon_{k}\right) \subseteq \Uparrow\left(p_{k}\right) \cap \max (P)$ and $O\left(v_{k}, T_{k}, \eta_{k}\right) \subseteq$ $\Uparrow\left(q_{k}\right)$. The sets $\left\{p_{k}: k<\omega\right\}$ and $\left\{q_{k}: k<\omega\right\}$ are directed subsets of $P$ and therefore have suprema in $P$. Therefore there exist $u_{*}, v_{*} \in \max (P)=$ $C_{\mathrm{p}}(X)$ with $p_{k} \ll p_{k+1} \sqsubseteq u_{*}$ and $q_{k} \ll q_{k+1} \sqsubseteq v_{*}$ for each $k$. Hence $u_{*} \in$ $\Uparrow\left(p_{k}\right) \subseteq O\left(u_{k}, S_{k}, \varepsilon_{k}\right)$ for each $k$ so that if $j \geq k$ and $x \in S_{k}$, then $\mid u_{*}(x)-$ $u_{k}(x)|=| u_{*}(x)-u_{j}(x) \mid<2^{-j}$. Consequently, $u_{*}(x)=u_{k}(x)$ whenever $x \in S_{k}$. Similarly, $v_{*}(x)=v_{k}(x)$ whenever $x \in S_{k}$. Therefore, $u_{*}(z)+v_{*}(z)=1$, while $u_{*}(x)+v_{*}(x)=0$ whenever $x \in S_{k}-\{z\}$. But then $y_{k} \in S_{k}$ gives $u_{*}\left(y_{k}\right)+v_{*}\left(y_{k}\right)=0$ for every $k$, while $u_{*}(z)+v_{*}(z)=1$. That is impossible because $u_{*}+v_{*} \in C_{\mathrm{p}}(X)$ and $z \in \operatorname{cl}\left(\left\{y_{k}: k<\omega\right\}\right)$.

Our next three lemmas show how to extend an acceptable 4-tuple in various ways. In combination, they show how to extend any fully acceptable 4-tuple to a larger fully acceptable 4-tuple.

LEMmA 4.2. Suppose $X$ is completely regular and pseudo-normal and that $(\beta, D, E, S)$ is an acceptable 4-tuple. Then there is an acceptable 4-tuple $\left(\beta+1, D^{+}, E^{+}, S^{+}\right)$with $D \subseteq D^{+}, E \subseteq E^{+}$, and $S \subseteq S^{+}$.

Proof. We know that $\beta<\kappa$ so that $|\beta| \leq \beta<\kappa=|\kappa|$, and that $y_{\alpha} \in S$ whenever $\alpha<\beta$. If $y_{\beta} \in S$, then $(\beta+1, D, E, S)$ is the required acceptable 4 -tuple, so assume $y_{\beta} \notin S$. Because $D$ and $E$ are bounded subsets of $P$, we fix any $u_{0}, v_{0} \in \max (P)$ with $D \subseteq \downarrow\left(u_{0}\right)$ and $E \subseteq \downarrow\left(v_{0}\right)$. Let $f_{0}=u_{0}$. Then $O\left(f_{0},\left\{z, y_{\beta}\right\}, 1\right)$ is a neighborhood of $f_{0}$ in $C_{\mathrm{p}}(X)=\max (P)$ so there is some $p_{0} \in P$ with $f_{0} \in \Uparrow\left(p_{0}\right) \cap \max (P) \subseteq O\left(f_{0},\left\{y_{\beta}, z\right\}, 1\right)$. There is a finite set $S_{0} \subseteq X$ and some $\varepsilon_{0}<2^{0}$ with $O\left(f_{0}, S_{0}, \varepsilon_{0}\right) \subseteq \Uparrow\left(p_{0}\right) \cap$ 
$\max (P) \subseteq O\left(f_{0},\left\{y_{\beta}, z\right\}, 1\right)$. Then $\left\{y_{\beta}, z\right\} \subseteq S_{0}$ and $\varepsilon_{0} \leq 1$. Next we use complete regularity of $X$ to modify $v_{0}$ on the finite set $S_{0}-S$. Because $\left|S_{0} \cup S\right|<\kappa, S_{0} \cup S$ is closed discrete. Then there is a function $g_{0} \in C_{\mathrm{p}}(X)$ with $g_{0}(x)=v_{0}(x)$ whenever $x \in S$ and $g_{0}(x)=-f_{0}(x)$ if $x \in S_{0}-S$. Then $O\left(g_{0}, S_{0}, \varepsilon_{0}\right)$ is a neighborhood of $g_{0}$ so there is a $q_{0} \in P$ with $g_{0} \in$ $\Uparrow\left(q_{0}\right) \cap \max (P) \subseteq O\left(g_{0}, S_{0}, \varepsilon_{0}\right)$. Find a finite set $T_{0}$ and a positive $\eta_{0}$ with $O\left(g_{0}, T_{0}, \eta_{0}\right) \subseteq \Uparrow\left(q_{0}\right) \cap \max (P) \subseteq O\left(g_{0}, S_{0}, \varepsilon_{0}\right)$. It follows that $S_{0} \subseteq T_{0}$ and $\eta_{0} \leq \varepsilon_{0}$. Note that if $x \in\left(S \cup S_{0}\right)-\{z\}$ then $u_{0}(x)+v_{0}(x)=0$ while $u_{0}(z)+v_{0}(z)=1$.

We will use a recursive construction. Suppose $k>0$ and for $0 \leq i<k$ we have finite sets $S_{i}$ and $T_{i}$, positive numbers $\varepsilon_{i}$ and $\eta_{i}$, and elements $p_{i}, q_{i} \in P$ that satisfy:

(R1) $\left\{y_{\beta}, z\right\} \subseteq S_{0} \subseteq T_{0} \subseteq \cdots \subseteq S_{k-1} \subseteq T_{k-1}$

(R2) $p_{i} \ll p_{j}$ and $q_{i} \ll q_{j}$ whenever $0 \leq i<j<k$;

(R3) $\eta_{i} \leq \varepsilon_{i}<2^{-i}$ whenever $0 \leq i<k$;

(R4) if $0 \leq i<j<k$ then $f_{j}(x)=f_{i}(x)$ for all $x \in S_{i}$ and $g_{j}(x)=g_{i}(x)$ for all $x \in T_{i}$;

(R5) $O\left(f_{i}, S_{i}, \varepsilon_{i}\right) \subseteq \Uparrow\left(p_{i}\right) \cap \max (P) \subseteq O\left(f_{i}, T_{i-1}, \eta_{i-1}\right)$ and $O\left(g_{i}, T_{i}, \eta_{i}\right)$ $\subseteq \Uparrow\left(q_{i}\right) \cap \max (P) \subseteq O\left(g_{i}, S_{i}, \varepsilon_{i}\right)$ whenever $0 \leq i<k$

(R6) if $x \in S_{i}-\{z\}$ then $f_{i}(x)+g_{i}(x)=0$, and $f_{i}(z)+g_{i}(z)=1$;

(R7) if $i<j<k$ and $x \in S_{k}$, then $f_{i}(x)=f_{j}(x)$ and $g_{i}(x)=g_{j}(x)$.

Using complete regularity of $X$, we define $f_{k}$ by modifying $f_{k-1}$ on the set $T_{k-1}-\left(S \cup S_{k-1}\right)$. Because $\left|T_{k-1} \cup S\right|<\kappa$, the set $T_{k-1} \cup S$ is closed discrete. Therefore there is some $f_{k} \in C_{\mathrm{p}}(X)$ with $f_{k}(x)=f_{k-1}(x)$ if $x \in S \cup S_{k-1}$, and $f_{k}(x)=-g_{k-1}(x)$ if $x$ is in the finite set $T_{k-1}-\left(S \cup S_{k-1}\right)$. Then

$$
O\left(f_{k}, T_{k-1}, \eta_{k-1}\right) \subseteq O\left(f_{k}, S_{k-1}, \varepsilon_{k-1}\right)=O\left(f_{k-1}, S_{k-1}, \varepsilon_{k-1}\right) \subseteq \Uparrow\left(p_{k-1}\right)
$$

because $S_{k-1} \subseteq T_{k-1}$ and $\eta_{k-1} \leq \varepsilon_{k-1}$. Therefore we can find some $p_{k} \in P$ with

$$
f_{k} \in \Uparrow\left(p_{k}\right) \cap \max (P) \subseteq O\left(f_{k}, T_{k-1}, \eta_{k-1}\right) \subseteq \Uparrow\left(p_{k-1}\right) .
$$

Lemma 2.2 allows us to choose $p_{k}$ with $p_{k-1} \ll p_{k}$. Then there is a finite set $S_{k} \subseteq X$ and a number $\varepsilon_{k} \in\left(0,2^{-k}\right)$ with $O\left(f_{k}, S_{k}, \varepsilon_{k}\right) \subseteq \Uparrow\left(p_{k}\right)$. Note that $T_{k-1} \subseteq S_{k}$ and $\varepsilon_{k} \leq \eta_{k-1}$.

We use complete regularity of $X$ to find some $g_{k} \in C_{\mathrm{p}}(X)$ with the property that for $x \in S \cup T_{k-1}, g_{k}(x)=g_{k-1}(x)$ and if $x$ is in the finite set $S_{k}-\left(S \cup T_{k-1}\right)$ then $g_{k}(x)=-f_{k}(x)$. Then $g_{k}(z)+f_{k}(z)=1$ and $g_{k}(x)+f_{k}(x)=0$ for each $x \in\left(S \cup S_{k}\right)-\{z\}$. Because $T_{k-1} \subseteq S_{k}$ and $\varepsilon_{k} \leq \eta_{k-1}$, we have

$$
O\left(g_{k}, S_{k}, \varepsilon_{k}\right) \subseteq O\left(g_{k}, T_{k-1}, \eta_{k-1}\right)=O\left(g_{k-1}, T_{k-1}, \eta_{k-1}\right) \subseteq \Uparrow\left(q_{k-1}\right),
$$


so that we can find some $q_{k} \in P$ with

$$
g_{k} \in \Uparrow\left(q_{k}\right) \cap \max (P) \subseteq O\left(g_{k}, S_{k}, \varepsilon_{k}\right) \subseteq \Uparrow\left(q_{k-1}\right) .
$$

Lemma 2.2 allows us to choose $q_{k}$ with $q_{k-1} \ll q_{k}$. Then there is a finite subset $T_{k} \subseteq X$ and a positive $\eta_{k}$ with

$$
O\left(g_{k}, T_{k}, \eta_{k}\right) \subseteq \Uparrow\left(q_{k}\right) \cap \max (P) \subseteq O\left(g_{k}, S_{k}, \varepsilon_{k}\right),
$$

from which it follows that $S_{k} \subseteq T_{k}$ and $\eta_{k} \leq \varepsilon_{k}$.

This recursion produces $S_{k}, T_{k}, f_{k}, g_{k}, \varepsilon_{k}, \eta_{k}, p_{k}$, and $q_{k}$ for each $k<\omega$. Note that $\bigcup\left\{S_{k}: k<\omega\right\}=\bigcup\left\{T_{k}: k<\omega\right\}$. Define $D^{+}=D \cup\left\{p_{k}: k<\omega\right\}$, $E^{+}=E \cup\left\{q_{k}: k<\omega\right\}$, and $S^{+}=S \cup \bigcup\left\{S_{k}: k<\omega\right\}$. Note that $S^{+}$is a closed, discrete subspace of $X$ because its cardinality is less than $\kappa$. We claim that $\left(\beta+1, D^{+}, E^{+}, S^{+}\right)$is an acceptable 4 -tuple. We will show that (A1) through (A7) are satisfied by the new 4-tuple.

Conditions (A1) and (A2) are clear because $\omega<\kappa$ by Lemma 4.1. To verify (A3) we will show that $D^{+}$is a bounded subset of $P$; the analogous assertion for $E^{+}$is proved similarly. If $x \in S$, then $f_{i}(x)=f_{j}(x)$ for all $i, j$. If $x \in S_{k}$ and if $j>k$, then $f_{j}(x)=f_{k}(x)$. Therefore, for each $x \in \bigcup\left\{S_{k}\right.$ : $k<\omega\}$, the real number $r_{k}=\lim \left\{f_{k}(x): k \rightarrow \omega\right\}$ exists. We now invoke the hypothesis that $X$ is completely regular and pseudo-normal. Lemma 2.3 gives us some function $u_{1} \in C_{\mathrm{p}}(X)=\max (P)$ with $u_{1}(x)=\lim \left\{f_{k}(x)\right.$ : $k \rightarrow \omega\}$ for each $x \in S^{+}$. Because $(\beta, D, E, S)$ is an acceptable 4-tuple and $u_{1}(x)=u_{0}(x)$ for all $x \in S$, we know that $D \subseteq \downarrow\left(u_{1}\right)$. Consider any $p_{k} \in D^{+}$. Because $u_{1}(x)=f_{k}(x)$ for each $x \in S_{k}$ we know that $u_{1} \in O\left(f_{k}, S_{k}, \varepsilon_{k}\right) \subseteq$ $\Uparrow\left(p_{k}\right)$ so that $p_{k} \ll u_{1}$. Hence $D^{+} \subseteq \downarrow\left(u_{1}\right)$, showing that $D^{+}$is a bounded subset of $P$, as required in (A3).

Because $(\beta, D, E, S)$ is given as an acceptable 4-tuple, condition (A4) holds for the set $D$, and because $p_{k} \ll p_{k+1}$ it also holds for all points of $D^{+}-D$. To verify (A5) we will use the function $u_{1}$ defined above, and any other $u_{2} \in C_{\mathrm{p}}(X)=\max (P)$ with $D^{+} \subseteq \downarrow\left(u_{2}\right)$. Because $D \subseteq D^{+}$condition (A5) for $(\beta, D, E, S)$ shows that $u_{2}(x)=u_{1}(x)$ for all $x \in S$. We also know that $u_{1}(x)=f_{k}(x)$ for each $x \in S_{k}$, because of the way that $u_{1}$ was defined. Fix some $x \in S_{k}$ and consider any $j>k$. We know that $f_{j}(x)=f_{k}(x)$. Also, $p_{j} \ll p_{j+1} \ll p_{j+2} \sqsubseteq u_{2}$ so that $u_{2} \in \Uparrow\left(p_{j+1}\right) \cap \max (P) \subseteq O\left(f_{j+1}, T_{j}, \eta_{j}\right) \subseteq$ $O\left(f_{j}, S_{j}, \varepsilon_{j}\right)$. Hence $\left|u_{2}(x)-f_{k}(x)\right|=\left|u_{2}(x)-f_{j}(x)\right|<\varepsilon_{j}<2^{-j}$ for each $j>k$, so that $u_{2}(x)=f_{k}(x)=u_{1}(x)$ as claimed.

To verify (A6), consider the function $u_{1}$ defined above and any other function $u_{2} \in C_{\mathrm{p}}(X)$ with $u_{2}(x)=u_{1}(x)$ for all $x \in S^{+}$. Because $(\beta, D, E, S)$ is an acceptable 4 -tuple we know that $D \subseteq \downarrow\left(u_{2}\right)$. It remains to show that $p_{k} \sqsubseteq u_{2}$. We note that if $x \in S_{k}$ then $u_{2}(x)=u_{1}(x)=f_{k}(x)$ so that $u_{2} \in O\left(f_{k}, S_{k}, \varepsilon_{k}\right) \subseteq \Uparrow\left(p_{k}\right)$, giving $p_{k} \ll u_{2}$ so that $p_{k} \sqsubseteq u_{2}$ as required. 
To verify (A7), suppose $u, v \in C_{\mathrm{p}}(X)$ with $D^{+} \subseteq \downarrow(u)$ and $E^{+} \subseteq \downarrow(v)$. Then $D \subseteq \downarrow(u)$ and $E \subseteq \downarrow(v)$ so that $u(z)+v(z)=1$ while $u(x)+v(x)=0$ for each $x \in S-\{z\}$. Consider any $x \in S^{+}-S$ and choose $k$ with $x \in S_{k}$. Then $u(x)=f_{k}(x)$ and $v(x)=g_{k}(x)$ so that recursion condition (R6) gives the desired conclusion.

Corollary 4.3. Suppose $X$ is completely regular. Then there is an acceptable 4-tuple $(1, D, E, S)$.

Proof. Part (A1) of the definition of acceptable 4-tuple requires that $z \in S$. Consequently, the 4 -tuple $(\beta, D, E, S)=(0, \emptyset, \emptyset, \emptyset)$ is not acceptable. However, the proof of Lemma 4.2 still applies and is designed in such a way that it could start with $(\beta, D, E, S)=(0, \emptyset, \emptyset, \emptyset)$ and produce an acceptable 4-tuple $\left(1,\left\{p_{k}: k<\omega\right\},\left\{q_{k}: k<\omega\right\}, \bigcup\left\{S_{k}: k<\omega\right\}\right)$ because the construction forces $z, y_{0} \in S_{0}$. Notice that we do not need the pseudo-normality hypothesis to get the continuous function that bounds $\left\{p_{k}: k<\omega\right\}$, because $\left\{p_{k}: k<\omega\right\}$ is a directed set in the domain $P$.

Lemma 4.4. Suppose $X$ is completely regular and pseudo-normal, and that $(\beta, D, E, S)$ is an acceptable 4-tuple. Fix $p, q \in D$ and $r, s \in E$. Then there is an acceptable 4-tuple $\left(\beta, D^{\prime}, E^{\prime}, S^{\prime}\right)$ with $D \subseteq D^{\prime}, E \subseteq E^{\prime}, S \subseteq S^{\prime}$ and with the property that some $d \in D^{\prime}$ and some $e \in E^{\prime}$ have $p, q \ll d$ and $r, s \ll e$.

Proof. Because the given 4-tuple is acceptable, we may choose $u_{*}, v_{*} \in$ $C_{\mathrm{p}}(X)=\max (P)$ with $D \subseteq \downarrow\left(u_{*}\right)$ and $E \subseteq \downarrow\left(v_{*}\right)$. Note that any functions $u, v \in C_{\mathrm{p}}(X)$ that agree with $u_{*}$ and $v_{*}$ respectively on the closed discrete set $S$ will also be upper bounds for $D$ and $E$, respectively, in $P$.

Let $u_{0}=u_{*}$. Because $d \in D$, condition (A4) gives $p^{\prime} \in D$ with $p \ll$ $p^{\prime} \sqsubseteq u_{0}$ so that $u_{0} \in \Uparrow(p)$. Similarly, $u_{0} \in \Uparrow(q)$ and $v_{*} \in \Uparrow(r) \cap \Uparrow(s)$. Choose $d_{0} \in P$ with $u_{0} \in \Uparrow\left(d_{0}\right) \subseteq \Uparrow(p) \cap \Uparrow(q)$. In the light of Lemma 2.2 we may assume that $p, q \ll d_{0}$. Choose a finite set $S_{0}$ and $\varepsilon_{0} \in\left(0,2^{0}\right)$ with $u_{0} \in O\left(u_{0}, S_{0}, \varepsilon_{0}\right) \subset \Uparrow\left(d_{0}\right)$. Because $X$ is completely regular and $S$ is closed in $X$, there is a function $v_{0} \in C_{\mathrm{p}}(X)$ with $v_{0}(x)=v_{*}(x)$ for each $x \in S$ and $v_{0}(x)=-u_{0}(x)$ for each $x$ in the finite set $S_{0}-S$. Then $v_{0} \in \Uparrow(r) \cap \Uparrow(s) \cap O\left(v_{0}, S_{0}, \varepsilon_{0}\right)$ so there is some $e_{0} \in P$ with

$$
v_{0} \in \Uparrow\left(e_{0}\right) \cap \max (P) \subseteq \Uparrow(r) \cap \Uparrow(s) \cap O\left(v_{0}, S_{0}, \varepsilon_{0}\right),
$$

and then some finite set $T_{0}$ and a positive $\eta_{0}$ with $v_{0} \in O\left(v_{0}, T_{0}, \eta_{0}\right) \subseteq$ $\Uparrow\left(e_{0}\right) \cap \max (P) \subseteq O\left(v_{0}, S_{0}, \varepsilon_{0}\right)$. It follows that $S_{0} \subseteq T_{0}$ and $\eta_{0} \leq \varepsilon_{0}$.

The set $S \cup S_{0}$ is closed and $T_{0}-\left(S \cup S_{0}\right)$ is finite. Because $X$ is completely regular there is a function $u_{1} \in C_{\mathrm{p}}(X)$ with $u_{1}(x)=u_{0}(x)$ for each $x \in S \cup S_{0}$ and $u_{1}(x)=-v_{0}(x)$ for each $x \in T_{0}-\left(S \cup S_{0}\right)$. Then $O\left(u_{1}, T_{0}, \eta_{0}\right) \subseteq O\left(u_{1}, S_{0}, \varepsilon_{0}\right)=O\left(u_{0}, S_{0}, \varepsilon_{0}\right) \subseteq \Uparrow\left(d_{0}\right)$ so there is some $d_{1} \in P$ with $u_{1} \in \Uparrow\left(d_{1}\right) \cap \max (P) \subseteq O\left(u_{1}, T_{0}, \eta_{0}\right)$. Lemma 2.2 allows us to choose 
$d_{1}$ with $d_{0} \ll d_{1}$. Then we have a finite set $S_{1}$ and a number $\varepsilon_{1} \in\left(0,2^{-1}\right)$ with $O\left(u_{1}, S_{1}, \varepsilon_{1}\right) \subseteq \Uparrow\left(d_{1}\right) \cap \max (P) \subseteq O\left(u_{1}, T_{0}, \eta_{0}\right)$ so that $T_{0} \subseteq S_{1}$ and $\varepsilon_{1} \leq \eta_{0}$.

Because the set $S_{1}-\left(S \cup T_{0}\right)$ is finite, there is a function $v_{1} \in C_{\mathrm{p}}(X)$ with $v_{1}(x)=v_{0}(x)$ for all $x \in S \cup T_{0}$ and $v_{1}(x)=-u_{1}(x)$ for all $x \in S_{1}-\left(S \cup T_{0}\right)$. Then $O\left(v_{1}, S_{1}, \varepsilon_{1}\right) \subseteq O\left(v_{1}, T_{0}, \eta_{0}\right)=O\left(v_{0}, T_{0}, \eta_{0}\right) \subseteq \Uparrow\left(e_{0}\right)$. This allows us to find $e_{1} \in P$ with $v_{1} \in \Uparrow\left(e_{1}\right) \cap \max (P) \subseteq O\left(v_{1}, S_{1}, \varepsilon_{1}\right) \subseteq \Uparrow\left(e_{0}\right)$ and with $e_{0} \ll e_{1}$. We find a finite $T_{1}$ and a positive $\eta_{1}$ with $O\left(v_{1}, T_{1}, \eta_{1}\right) \subseteq$ $\Uparrow\left(e_{1}\right) \cap \max (P) \subseteq O\left(v_{1}, S_{1}, \varepsilon_{1}\right)$. Then $S_{1} \subseteq T_{1}$ and $\eta_{1} \leq \varepsilon_{1}$.

This recursion produces $u_{k}, v_{k}, S_{k}, T_{k}, \varepsilon_{k}$, and $\eta_{k}$ just as in Lemma 4.2, and just as in that lemma, if we let $D^{\prime}=D \cup\left\{d_{k}: k<\omega\right\}, E^{\prime}=$ $E \cup\left\{e_{k}: k<\omega\right\}$, and $S=S \cup \bigcup\left\{S_{k}: k<\omega\right\}$ we obtain an acceptable 4 -tuple $\left(\beta, D^{\prime}, E^{\prime}, S^{\prime}\right)$. The hypothesis of pseudo-normality is used when we invoke Lemma 2.3 in the proof that the sets $D^{\prime}$ and $E^{\prime}$ are bounded subsets of $P$. Because $p, q \ll d_{0} \in D^{\prime}$ and $r, s \ll e_{0} \in E^{\prime}$, the lemma is proved.

Recall that an acceptable 4-tuple $(\beta, D, E, S)$ is fully acceptable provided the sets $D$ and $E$ are directed subsets of $P$, rather than just bounded in $P$. The next lemma is the first in this section that seems to require the full force of the normality of $X$.

Lemma 4.5. Suppose $X$ is normal and that $(\beta, D, E, S)$ is an acceptable 4-tuple. Then there is a fully acceptable 4-tuple $\left(\beta, D^{*}, E^{*}, S^{*}\right)$ with the property that $D \subseteq D^{*}, E \subseteq E^{*}$, and $S \subseteq S^{*}$.

Proof. Using transfinite induction, we will show that there is an acceptable 4-tuple $\left(\beta, D^{(1)}, E^{(1)}, S^{(1)}\right)$ with $D \subseteq D^{(1)}, E \subseteq E^{(1)}$, and $S \subseteq S^{(1)}$, and with the property that for any $p, q \in \bar{D}$ and any $r, s \in E$, some $d \in D^{(1)}$ has $p, q \ll d$ and some $e \in E^{(1)}$ has $r, s \ll e$. Once that part of the proof is completed, we will apply it recursively to obtain acceptable 4-tuples $\left(\beta, D^{(k)}, E^{(k)}, S^{(k)}\right)$ with $D^{(k)} \subseteq D^{(k+1)}, E^{(k)} \subseteq E^{(k+1)}$, and $S^{(k)} \subseteq S^{(k+1)}$ so that each pair $p, q \in D^{(k)}$ has some $d \in D^{(k+1)}$ with $p, q \ll d$ and such that an analogous statement holds for $E^{(k)}$ and $E^{(k+1)}$. Then we let $D^{*}=\bigcup\left\{D^{(k)}: k<\omega\right\}, E^{*}=\bigcup\left\{E^{(k)}: k<\omega\right\}$, and $S^{*}=\bigcup\left\{S^{(k)}: k<\omega\right\}$. Then the sets $D^{*}$ and $E^{*}$ are directed subsets of $P$ so that the resulting 4 -tuple $\left(\beta, D^{*}, E^{*}, S^{*}\right)$ is fully acceptable, as required.

To construct $D^{(1)}$ and $E^{(1)}$, we well order the sets $D \times D$ and $E \times E$ as $\left\{\left(p_{\alpha}, q_{\alpha}\right): \alpha<\max (|\beta|, \omega)\right\}$ and $\left\{\left(r_{\alpha}, s_{\alpha}\right): \alpha<\max (|\beta|, \omega)\right\}$ respectively. Repetitions are allowed, if necessary. Let $D_{0}=D, E_{0}=E$, and $S_{0}=S$.

We use Lemma 4.4 to extend $\left(\beta, D_{0}, E_{0}, S_{0}\right)$ to an acceptable 4 -tuple $\left(\beta, D_{1}, E_{1}, S_{1}\right)$ such that some $d \in D_{1}$ and some $e \in E_{1}$ have $p_{0}, q_{0} \ll d$ and $r_{0}, s_{0} \ll e$. 
Suppose $\gamma<\max (|\beta|, \omega)$ and that for each $\alpha<\gamma$ we have an acceptable 4 -tuple $\left(\beta, D_{\alpha}, E_{\alpha}, S_{\alpha}\right)$ with the properties that

$\left(a_{\gamma}\right)$ if $\delta<\eta<\gamma$ then $D_{\delta} \subseteq D_{\eta}, E_{\delta} \subseteq E_{\eta}$, and $S_{\delta} \subseteq S_{\eta}$;

$\left(b_{\gamma}\right)$ if $\delta<\eta<\gamma$ then some $d \in D_{\eta}$ and $e \in E_{\eta}$ have $p_{\delta}, q_{\delta} \ll d$ and $r_{\delta}, s_{\delta} \ll e$.

In case $\gamma=\eta+1$, use Lemma 4.4 to extend $\left(\beta, D_{\eta}, E_{\eta}, S_{\eta}\right)$ to $\left(\beta, D_{\eta+1}\right.$, $\left.E_{\eta+1}, S_{\eta+1}\right)$. The remaining case is where $\gamma$ is a limit ordinal. Define $D_{\gamma}=$ $\bigcup\left\{D_{\eta}: \eta<\gamma\right\}, E_{\gamma}=\bigcup\left\{E_{\eta}: \eta<\gamma\right\}$ and $S_{\gamma}=\bigcup\left\{S_{\eta}: \eta<\gamma\right\}$. We claim that $\left(\beta, D_{\gamma}, E_{\gamma}, S_{\gamma}\right)$ is an acceptable 4-tuple. There are several things to verify. (A1) clearly holds. Consider (A2). Each $D_{\eta}$ has cardinality less than or equal to $\max (|\beta|, \omega)$ and there are at most $|\gamma|$-many of them, where $|\gamma| \leq \gamma<\max (|\beta|, \omega)$, so that $\left|D_{\gamma}\right| \leq \max (|\beta|, \omega)$. Similarly, $E_{\gamma}$ and $S_{\gamma}$ have cardinality less than or equal to $\max (|\beta|, \omega)$.

Next we show that $D_{\gamma}$ is a bounded subset of $P$. Because $S_{\gamma}$ has cardinality less than $\kappa, S_{\gamma}$ is closed an discrete in $X$. For each $\alpha<\gamma$ choose some $u_{\alpha} \in C_{\mathrm{p}}(X)$ with $D_{\alpha} \subseteq \downarrow\left(u_{\alpha}\right)$. Note that if $\delta<\eta<\gamma$, then $D_{\delta} \subseteq$ $D_{\eta} \subseteq \downarrow\left(u_{\eta}\right)$. Property (A5) applied to $u_{\delta}$ and $u_{\eta}$ shows that $u_{\eta}(x)=u_{\delta}(x)$ for each $x \in S_{\delta}$. Therefore for each $x \in S_{\gamma}, \lim \left\{u_{\alpha}(x): \alpha \rightarrow \gamma\right\}$ is a real number so that the rule $x \rightarrow \lim \left\{u_{\alpha}(x): \alpha \rightarrow \gamma\right\}$ gives a function from the closed discrete subspace $S_{\gamma}$ into the space of real numbers. Normality of $X$ provides a function $u_{\gamma} \in C_{\mathrm{p}}(X)$ that has $u_{\gamma}(x)=\lim \left\{u_{\eta}(x): \eta \rightarrow \gamma\right\}$, and this $u_{\gamma} \in \max (P)$ is an upper bound for each set $D_{\eta}$ with $\eta<\gamma$. Therefore $u_{\gamma}$ is also an upper bound for $D_{\gamma}$. Similarly, $E_{\gamma}$ is a bounded subset of $P$.

Properties (A4)-(A7) hold for $D_{\gamma}$ and $E_{\gamma}$ because they hold for each $D_{\eta}$ and $E_{\eta}$ with $\eta<\gamma$.

The above transfinite recursion gives acceptable 4-tuples $\left(\beta, D_{\gamma}, E_{\gamma}, S_{\gamma}\right)$ for each $\gamma<\max (|\beta|, \omega)$ with the properties $\left(a_{\gamma}\right)$ and $\left(b_{\gamma}\right)$. Because $\max (|\beta|, \omega)$ is a limit ordinal less than $\kappa$, we obtain the desired extension $\left(\beta, D^{(1)}, E^{(1)}, S^{(1)}\right)$ by letting $D^{(1)}=\bigcup\left\{D_{\gamma}: \gamma<\max (|\beta|, \omega)\right\}, E^{(1)}=$ $\bigcup\left\{E_{\gamma}: \gamma<\max (|\beta|, \omega)\right\}$ and $S^{(1)}=\bigcup\left\{S_{\gamma}: \gamma<\max (|\beta|, \omega)\right\}$. Then, as explained in the proof's first paragraph, we obtain the desired fully acceptable 4 -tuple.

COROLlary 4.6. If $X$ is normal, then there is a fully acceptable 4-tuple $\left(1, D^{*}, E^{*}, S^{*}\right)$.

Proof. By Corollary 4.3, there is an acceptable 4-tuple (1, D, E, S). Now apply Lemma 4.5 to that acceptable 4 -tuple to produce the required fully acceptable $\left(1, D^{*}, E^{*}, S^{*}\right)$. 
Lemma 4.7. Suppose $X$ is normal. Let $\Phi$ be a maximal chain in the nonempty poset $(\Psi, \preceq)$. For any 4 -tuple in $\Phi$, let $\pi_{1}$ be first-coordinate projection. Then the set $\pi_{1}[\Phi]=\left\{\pi_{1}(\beta, D, E, S):(\beta, D, E, S) \in \Phi\right\}$ is cofinal in $\kappa$.

Proof. Write $I=\pi_{1}[\Phi]$ and $\lambda=\sup (I)$. Then $\lambda \leq \kappa$. If $\lambda=\kappa$ there is nothing to prove. For contradiction, suppose $\lambda<\kappa$. If $\lambda \in I$ then choose $(\lambda, D, E, S) \in \Phi$. Because $X$ is completely regular and pseudo-normal we may apply Lemma 4.2 to $(\lambda, D, E, S)$ to produce an acceptable 4 -tuple $\left(\lambda+1, D^{\prime}, E^{\prime}, S^{\prime}\right)$ with $D \subseteq D^{\prime}, E \subseteq E^{\prime}$, and $S \subseteq S^{\prime}$. Then, because $X$ is normal, we may apply Lemma 4.5 to $\left(\lambda+1, D^{\prime}, E^{\prime}, S^{\prime}\right)$ to produce a fully acceptable 4-tuple $\left(\lambda+1, D^{*}, E^{*}, S^{*}\right)$ with $D \subseteq D^{\prime} \subseteq D^{*}, E \subseteq E^{\prime} \subseteq E^{*}$, and $S \subseteq S^{\prime} \subseteq E^{*}$. But then $\Phi \cup\left\{\left(\lambda+1, D^{*}, E^{*}, S^{*}\right)\right\}$ is a chain in $\Psi$ that is strictly larger than $\Phi$, and that is impossible. Hence $\lambda \notin I$. Therefore $\lambda$ is an infinite limit ordinal.

Because $\Phi$ is a chain in $(\Psi, \preceq)$, we may index the elements of $\Phi$ in a monotonic way using their first coordinates. This allows us to write $\Phi=$ $\left\{\left(\beta, D_{\beta}, E_{\beta}, S_{\beta}\right): \beta \in I\right\}$ with $D_{\beta} \subseteq D_{\gamma}, E_{\beta} \subseteq E_{\gamma}$, and $S_{\beta} \subseteq S_{\gamma}$ whenever $\beta<\gamma$ are in $I$. Let $D^{* *}=\bigcup\left\{D_{\beta}: \beta \in I\right\}, E^{* *}=\bigcup\left\{E_{\beta}: \beta \in I\right\}$ and $S^{* *}=\bigcup\left\{S_{\beta}: \beta \in I\right\}$. We claim that $\left(\lambda, D^{* *}, E^{* *}, S^{* *}\right)$ is a fully acceptable 4-tuple.

Because $\lambda<\kappa$, property (A1) is immediate. To prove (A2) consider $D^{* *}$. This set is a union of at most $|\lambda|$-many sets, each of cardinality less than or equal to $\max (|\gamma|, \omega)$ where $\gamma<\lambda<\kappa$. Hence each of the sets $D_{\beta}$ used to construct $D^{* *}$ has cardinality less than $|\lambda|$ and there are at most $|\lambda|$-many of them, so that $D^{* *}$ has cardinality less than or equal to $|\lambda|=\max (|\lambda|, \omega)$. The same statement holds for $E^{* *}$ and $S^{* *}$. Property (A3) holds because $D^{* *}$ and $E^{* *}$ are unions of chains of directed subsets of $P$, and therefore both $D^{* *}$ and $E^{* *}$ are directed subsets of $P$.

Properties (A4)-(A7) follow directly from the definitions of $D^{* *}, E^{* *}$ and $S^{* *}$ as unions of chains of acceptable 4 -tuples. But now we contradict maximality of $\Phi$ because $\left(\lambda, D^{* *}, E^{* *}, S^{* *}\right) \in \Psi$ is strictly above every member of $\Phi$.

At this point the reader will see that all of the lemmas needed in the previous section have been established.

5. What to do without normality. In the results of the previous section, only Lemma 4.5, Corollary 4.6, and Lemma 4.7 used the normality hypothesis. In those three cases, normality was the key to extending a certain function defined on a possibly uncountable closed discrete subset of $X$ to be a continuous function on all of $X$. We do not know how to get around this problem, so we ask 
QUESTION 5.1. Suppose $X$ is completely regular and pseudo-normal. If $C_{\mathrm{p}}(X)$ is domain representable, must $X$ be discrete? Suppose $X$ is completely regular but not discrete. Can $C_{\mathrm{p}}(X)$ be domain representable?

As a partial answer to that question, we can prove:

Proposition 5.2. Suppose $X$ is completely regular and pseudo-normal. Then $C_{\mathrm{p}}(X)$ is Scott-domain representable if and only if $X$ is discrete.

Proof. As before, it is enough to prove that if $C_{\mathrm{p}}(X)$ is Scott-domain representable, then $X$ is discrete. Suppose $X$ is not discrete and yet $C_{\mathrm{p}}(X)$ is represented by the Scott domain $(P, \sqsubseteq)$. Let $\kappa$ be the smallest cardinal such that some subset $Y \subseteq X$ has cardinality $\kappa$ and is not closed. Well order $Y=\left\{y_{\alpha}: \alpha<\kappa\right\}$. Fix a limit point $z \in \operatorname{cl}(Y)-Y$. Define an acceptable 4-tuple as before and let $\Psi^{\prime}$ be the collection of all acceptable 4 -tuples. Then Corollary 4.3 shows that $\Psi^{\prime} \neq \emptyset$. Let $\preceq$ be the partial order used in the previous section and let $\Phi^{\prime}$ be a maximal chain in $\Psi^{\prime}$. Write $I^{\prime}=\pi_{1}\left[\Phi^{\prime}\right]$ and write $\Phi^{\prime}=\left\{(\beta, D, E, S): \beta \in I^{\prime}\right\}$. We claim that $I^{\prime}$ is cofinal in $\kappa$. If not, then the ordinal $\lambda^{\prime}=\sup \left(I^{\prime}\right)$ has $\lambda^{\prime}<\kappa$. If $\lambda^{\prime} \in I^{\prime}$, choose $\left(\lambda^{\prime}, D, E, S\right) \in \Phi^{\prime}$ and use Lemma 4.2 to extend $\left(\lambda^{\prime}, D, E, S\right)$ by one step to an acceptable 4 -tuple $\left(\lambda^{\prime}+1, D^{\prime}, E^{\prime}, S^{\prime}\right)$. But that is impossible because $\Phi^{\prime}$ is a maximal chain in $\Psi^{\prime}$, so $\lambda^{\prime} \notin I^{\prime}$. Then $\lambda^{\prime}$ is an infinite limit ordinal. Let $D^{\prime}=\bigcup\left\{D_{\beta}: \beta \in I^{\prime}\right\}, E^{\prime}=\bigcup\left\{E_{\beta}: \beta \in I^{\prime}\right\}$, and $S^{\prime}=\bigcup\left\{S_{\beta}: \beta \in I^{\prime}\right\}$. We claim that $D^{\prime}$ is a bounded subset of $P$.

For each $\beta \in I^{\prime}$ we know that $D_{\beta}$ is a bounded subset of $P$ so that, $P$ being a Scott domain, $\sup \left(D_{\beta}\right) \in P$. Note that if $\beta<\gamma$ both belong to $I^{\prime}$ then $D_{\beta} \subseteq D_{\gamma}$ so that $\sup \left(D_{\beta}\right) \sqsubseteq \sup \left(D_{\gamma}\right)$. Hence the set $\left\{\sup \left(D_{\beta}\right): \beta \in I^{\prime}\right\}$ is a directed subset of $P$ (in fact, a chain in $P$ ), so that because $P$ is a domain, $\sup \left(\left\{\sup \left(D_{\beta}\right): \beta \in I^{\prime}\right\}\right) \in P$, and that element of $P$ is an upper bound in $P$ for the set $D^{\prime}$. Similarly, $E^{\prime}$ is a bounded subset of $P$. (Note that we avoided the use of normality.) Because $\lambda^{\prime}$ is a limit ordinal, the other properties of an acceptable 4-tuple hold for $\left(\lambda^{\prime}, D^{\prime}, E^{\prime}, S^{\prime}\right)$ precisely because they hold for the members of $\Phi^{\prime}$ out of which $\left(\lambda^{\prime}, D^{\prime}, E^{\prime}, S^{\prime}\right)$ was constructed. But then $\Phi^{\prime} \cup\left\{\left(\lambda^{\prime}, D^{\prime}, E^{\prime}, S^{\prime}\right)\right\}$ is a chain in $\Psi^{\prime}$ that is strictly larger than $\Phi^{\prime}$, and that is impossible. Therefore the set $I^{\prime}$ is cofinal in $\kappa$.

For each $\beta \in I^{\prime}, \sup \left(D_{\beta}\right) \in P$ because $D_{\beta}$ is a bounded subset of the Scott domain $P$, and the set $\left\{\sup \left(D_{\beta}\right): \beta \in I^{\prime}\right\}$ is a directed subset (in fact, a chain) in the domain $P$. Hence there is some element $u^{*} \in \max (P)=$ $C_{\mathrm{p}}(X)$ that is an upper bound for $\left\{\sup \left(D_{\beta}\right): \beta \in I^{\prime}\right\}$. Similarly, there is some $v^{*} \in \max (P)=C_{\mathrm{p}}(X)$ that is an upper bound for the directed set $\left\{\sup \left(E_{\beta}\right): \beta \in I^{\prime}\right\}$.

Consider any $y_{\alpha} \in Y$. Because $I^{\prime}$ is cofinal in $\kappa$, some $\beta \in I^{\prime}$ has $\alpha<\beta$. Because $\sup \left(D_{\beta}\right) \sqsubseteq u^{*}, D_{\beta} \subseteq \downarrow\left(u^{*}\right)$, Similarly, $E_{\beta} \subseteq \downarrow\left(v^{*}\right)$. Therefore 
$u^{*}\left(y_{\alpha}\right)+v^{*}\left(y_{\alpha}\right)=0$, while $u^{*}(z)+v^{*}(z)=1$. As before, that is impossible because $u^{*}+v^{*}$ is continuous and $z \in \operatorname{cl}(Y)-Y$.

\section{References}

[1] H. Bennett and D. Lutzer, Domain-representability of certain complete spaces, Houston J. Math., to appear.

[2] G. Gierz, H. Hofmann, K. Keimel, J. D. Lawson, M. Mislove and D. S. Scott, Continuous Lattices and Domains, Encyclopedia Math. Appl. 93, Cambridge Univ. Press, Cambridge, 2003.

[3] L. Gillman and M. Jerison, Rings of Continuous Functions, Grad. Texts in Math. 43, Springer, New York, 1976.

[4] J. de Groot, Subcompactness and the Baire category theorem, Indag. Math. 25 (1963), 761-767.

[5] D. Lutzer and R. McCoy, Category in function spaces, I, Pacific J. Math. 90 (1980), $145-168$.

[6] D. Lutzer, J. van Mill and V. Tkachuk, Amsterdam properties of $C_{\mathrm{p}}(X)$ imply discreteness of $X$, Canad. Math. Bull., to appear.

[7] K. Martin, Topological games in domain theory, Topology Appl. 129 (2001), 177-186.

[8] K. Martin, M. Mislove and G. M. Reed, Topology and domain theory, in: Recent Progress in General Topology II, M. Hušek and J. van Mill (eds.), Elsevier, Amsterdam, 2002, 371-394.

Mathematics Department

Texas Tech University

Lubbock, TX 79409, U.S.A.

E-mail: harold.bennett@ttu.edu
Mathematics Department College of William and Mary Williamsburg, VA 23187-8795, U.S.A. E-mail: lutzer@math.wm.edu

Received 16 March 200\%;

in revised form 18 March 2008 